\title{
Quality of Life, Social Support, Coping Strategies, and Psychiatric Morbidity in Patients with Rheumatoid Arthritis
}

\author{
Naresh Nebhinani ${ }^{1}$ Surendra Kumar Mattoo ${ }^{2,3}$ \\ ${ }^{1}$ Department of Psychiatry, All India Institute of Medical Science, \\ Jodhpur, Rajasthan, India \\ 2 Department of Psychiatry, Postgraduate Institute of Medical \\ Education and Research (PGIMER), Chandigarh, India \\ ${ }^{3}$ Newcastle North East Community Treatment Team, Cumbria, \\ Northumberland, Tyne and Wear NHS Foundation Trust, Molineux \\ NHS Centre, Newcastle-upon-Tyne, United Kingdom \\ ${ }^{4}$ Department of Immunology, Postgraduate Institute of Medical \\ Education and Research (PGIMER), Chandigarh, India \\ ${ }^{5}$ Adventist Medical Center, Portland, Oregon, United States
}

\author{
Ajay Wanchu $u^{4,5}$
}

J Neurosci Rural Pract 2022;13:119-122.

\section{Abstract \\ Keywords \\ - rheumatoid arthritis \\ - quality of life \\ - coping \\ - social support \\ - psychiatric disorders}

Address for correspondence Naresh Nebhinani, MD, DNB, Department of Psychiatry, All India Institute of Medical Science, Jodhpur, Rajasthan 342005, India (e-mail: drnaresh_pgi@yahoo.com).

Background and Objectives Patients with rheumatoid arthritis (RA) have greater psychological morbidity, despite that research in this area is scarce from developing countries. This study was aimed to assess the association of quality of life, social support, coping strategies, and psychological morbidity in patients with RA.

Materials and Methods In this cross-sectional study, 40 patients with RA, who were not receiving steroids or disease modifying antirheumatic drugs, were recruited through purposive sampling. Social support questionnaire, coping strategy check list, and World Health Organization quality of life-BREF (WHOQOL-BREF) were administered to assess social support, coping, and quality of life, respectively.

Results More than half of the patients had psychiatric disorders (60\%), with depression being the commonest disorder (52.5\%). Internalization coping and disease severity indicators like tender joints counts, swollen joints counts, pain, and disease activity were found as significant predictors for psychiatric disorders, while externalization coping, quality of life (all domains), and physical functions were found to protect against psychiatric morbidity.

Conclusions Coping, quality of life, disease severity, and physical functions predicted the psychiatric disorders in RA. Multipronged interventions to enhance quality of life with promoting adaptive coping and timely treatment may further improve their mental health and overall disease course.

published online January 13, 2022
DOI https://doi.org/ $10.1055 / \mathrm{s}-0041-1742137$. ISSN 0976-3147. (c) 2022. Association for Helping Neurosurgical Sick People. All rights reserved.

This is an open access article published by Thieme under the terms of the Creative Commons Attribution-NonDerivative-NonCommercial-License, permitting copying and reproduction so long as the original work is given appropriate credit. Contents may not be used for commercial purposes, or adapted, remixed, transformed or built upon. (https://creativecommons.org/ licenses/by-nc-nd/4.0/)

Thieme Medical and Scientific Publishers Pvt. Ltd., A-12, 2nd Floor, Sector 2, Noida-201301 UP, India 


\section{Introduction}

Patients with rheumatoid arthritis (RA) have greater psychological morbidity especially depression and anxiety, along with greater disease activity, functional impairment, pain, and poor social support. ${ }^{1,2} \mathrm{~A}$ recent systematic review and meta-analysis reported lower quality of life (QOL) in RA with increasing age in female subjects. ${ }^{3}$ Maladaptive coping and other psychosocial factors influence individual's help-seeking behavior and also contribute to patient-related delay in initiating the treatment and negatively affect illness course and outcome. ${ }^{4}$ Despite that psychological health and coping of individuals have got lesser clinical attention, as reported in a systematic review of patient reported outcomes studies in RA. ${ }^{5}$ Psychological disorders are underdiagnosed and often remain untreated, which further contribute to deleterious impact on all health outcomes of RA.

Clinician's awareness about bidirectional relationship between psychosocial issues and RA through biopsychosocial approach may aid in thorough evaluation and comprehensive care to reduce disease burden. Psychosocial factors in RA related research are scarce in developing countries. Therefore, the present study aimed to assess the QOL, social support, coping strategies, and their association with psychiatric morbidity in patients with RA.

\section{Methods}

\section{Subjects}

The study was conducted at immunology outpatient setting, Postgraduate Institute of Medical Education and Research, Chandigarh, a tertiary care hospital in North India. The research had received ethics clearance from the Institutional Ethics Committee.

The sample comprised of 40 patients with RA (severe) not receiving steroids or disease-modifying antirheumatic drugs (DMARDs). Subjects aged between 18 and 55 years, who were able to read and write Hindi or English language, and who provided informed consent, were included and the subjects with any other comorbid medical disorder were excluded. Diagnosis of RA (severe) was made as per 1987 ACR (American College of Rheumatology) Revised Criteria for classification of RA and accordingly clinical details were ascertained about tender or swollen joint counts, assessment of pain, disease activity, and physical functions. Subsequently, assessment for psychiatric disorder was done through structured clinical interview by trained psychiatrist and diagnosis was made as per International Classification of Disease-10th revision and following self-administered tools were administered.

Social Support Questionnaire was used to assess perceived social support. Based on the Pollack and Harris scale, ${ }^{6}$ an Indian adaptation of scale was used, which has high testretest reliability $(r=0.91)$ and concurrent validity. ${ }^{7}$

Coping Strategy Check List assessed the strategies to cope with the stressful situations and further classified into denial, internalization, externalization, emotional outlet, and anger coping. ${ }^{8}$ Hindi translation of scale with Cronbach's $\alpha$ of 0.64 was used. ${ }^{9}$
World Health Organization Quality of Life-BREF was used to assess QOL in physical, psychological, social relationships, and environment domain. It has been shown to have good test-retest reliability, discriminant and content validity at several international collaborative studies. ${ }^{10}$

\section{Statistical Analyses}

Statistical Package for the Social Sciences (SPSS) version 21.0 for Windows (Chicago, Illinois, United States) was used for descriptive and inferential analysis. Correlates of psychiatric morbidity were assessed through correlational analysis and binomial logistic regression.

\section{Results}

\section{Demographic Details}

In 40 patients with RA, mean age was 43.5 years and mean education was 9 years. Majority of them were females (75\%), married (82.5\%), unemployed or housewife (72.5\%), Hindu by religion (82.5\%), and from nuclear family (82.5\%). Around half of the patients were residing in urban locality (55\%). The mean duration of RA was 83.37 months (i.e., $\sim 7$ years).

\section{Psychosocial Profile}

Psychiatric disorder was present in 60\% individuals and most common disorder was depression (52.5\%), followed by adjustment disorder (5\%) and panic disorder (2.5\%).

Mean social support score was 48.8 . Total coping score was 5.02 and among coping strategies, denial had the highest score, followed by internalization. In QOL domains, environment domain had maximum score, followed by social relationship domain and physical domain had the lowest score. Each domain of QOL had significant correlation with other domains and total score of QOL $(r=0.520-0.921, p<0.001)(-$ Table 1$)$.

\section{Association of Coping, Social Support, and QOL with Demographic and Clinical Variables}

Male and female subjects had comparable scores on disease severity, coping, social support, QOL, and psychiatric morbidity. However, older subjects had lesser anger coping $(r=-0.318$, $p=0.04)$. More educated subjects had greater physical QOL $(r=0.316, p=0.04)$. Income was protective against disease severity, as it was negatively associated with disease severity indicators-tender joints counts, swollen joints counts, and patient's assessment of pain $(r=-0.321$ to $-0.412, p<0.05)$. Patients with longer duration of illness had lower score of externalization coping $(r=-0.333, p=0.03)$.

All the disease severity indicators (tender or swollen joints, pain, and disease activity) were negatively associated with QOL ( $r=-0.346$ to $-0.675, p<0.01)$. Patient's assessment of physical functions was positively associated with physical, psychological, and environment domains of QOL $(r=0.450-0.570, p<0.01)$.

Subjects with better social support had lower internalization coping $(r=-0.588, p=0.001)$ and had greater social relationship QOL score $(r=0.629, p<0.001)$. Subjects with internalization coping had poor QOL $(r=-0.418-0.547, p<0.01)$ and greater disease severity indicators $(r=0.361-0.522, p<0.05)$. 
Table 1 Psychosocial profile

\begin{tabular}{|l|l|}
\hline Variable & Frequency/\%/mean (SD) \\
\hline Psychiatric disorder-present $^{\mathrm{a}}$ & $24(60)$ \\
\hline $\begin{array}{l}\text { Social support } \\
\text { questionnaire-total score }\end{array}$ & $48.82(8.32)$ \\
\hline Coping skills & $1.90(1.03)$ \\
\hline Denial & $1.85(1.42)$ \\
\hline Internalization & $0.45(0.74)$ \\
\hline Externalization & $0.45(0.55)$ \\
\hline Emotional outlet & $0.37(0.66)$ \\
\hline Anger & $5.02(1.76)$ \\
\hline Total coping score & \\
\hline QOL & $10.65(2.57)$ \\
\hline Physical & $10.85(2.40)$ \\
\hline Psychological & $11.96(2.64)$ \\
\hline Social relationship & $12.01(1.39)$ \\
\hline Environment & $45.48(7.82)$ \\
\hline Total QOL score &
\end{tabular}

Abbreviations: QOL, quality of life; SD, standard deviation.

a Depression-21 (52.5\%), adjustment disorder-2 (5\%), panic disorder1 (2.5\%).

Subjects with psychiatric disorder more commonly used internalization coping ( 2.33 vs. $1.12, p=0.006)$ and less commonly used externalization coping ( 0.25 vs. $0.75, p=0.028)$, and they had lesser social support ( 46.87 vs. $51.75, p=0.06$ ) compared with subjects without psychiatric disorders.

Subjects with psychiatric disorder had greater disease severity (tender joints counts [40.08 vs. 23.18], swollen joints counts [21.75 vs. 7.0$]$, pain [6.79 vs. 3.50], and disease activity [7.08 vs. 4.81] and lesser QOL total score [41.15 vs. 51.97] and QOL domain score-physical [9.16 vs. 12.89], psychological [9.75 vs. 12.5], social relationship [10.88 vs. 13.58], and environment domain [11.35 vs. 13.0 ], $p<0.001$ ), compared with subjects without psychiatric disorders.

\section{Psychosocial Correlates of Psychiatric Disorders}

On binomial logistic regression analysis with individual independent variable, internalization coping (odds ratio $[\mathrm{OR}]=2.067)$, and disease severity indicators-tender joints counts $(O R=1.153)$, swollen joints counts $(O R=1.210)$, pain $(O R=2.178)$, and disease activity $(O R=2.316)$ were found as significant predictors for psychiatric disorders. On the other side, externalization coping $(\mathrm{OR}=0.391), \mathrm{QOL}$ (all domains) $(\mathrm{OR}=0.184-0.474)$, and patient's assessment of physical functions ( $\mathrm{OR}=0.657$ ) were found to protect against psychiatric morbidity.

\section{Discussion}

Index study assessed association of QOL, social support, coping strategies with psychiatric morbidity in patients with RA in north India. In line with earlier study, ${ }^{2}$ greater proportion of the patients (60\%) had psychiatric disorders and it was associated with pain, functional impairment, and disease severity.

Coping is conceptualized as thoughts or behaviors that person employs to deal with stressful situations. ${ }^{11}$ In index study, denial had greater scores followed by internalization coping. In line with recent study, ${ }^{12}$ we also found association of coping with age, duration of illness, disease severity indicators, as anger coping was less reported in older patients, greater externalization coping was used in earlier course of RA, and more use of internalization coping was seen with greater disease severity. In index study, internalization coping (to increase the risk) and externalization coping (to reduce the risk) were significant predictors for psychiatric disorders.

QOL is a multidimensional concept, incorporating subjective report of individual's perceptions of positive and negative aspects of life. ${ }^{10}$ In index study, highest QOL score was seen in environment domain followed by social relationship domain and similar to earlier study, ${ }^{13}$ we found lowest QOL score in physical domain.

Similar to earlier studies, ${ }^{13-16}$ we reported positive association of QOL with education, social support, and physical functions, negative association of QOL with pain, disease activity, maladaptive coping (internalization), and psychiatric morbidity and no association was found with age, gender, and duration of illness. On the contrary, other studies ${ }^{3,14}$ reported lower QOL in female and older patients.

In line with other studies, ${ }^{3,17}$ our patients with psychiatric disorders had lower QOL and QOL domains and total score were significant predictor for psychiatric morbidity.

Poor social support, QOL, and use of maladaptive coping can be precursor or consequence of psychiatric disorders. Each domain of QOL correlates significantly with other domains of QOL, and similarly social support and coping also affect QOL; therefore, these psychosocial factors affect psychiatric morbidity and disease course.

The present study had several limitations like small sample size, noninclusion of patients on steroids or DMARDs, recruitment of all patients from a single center and due to cross-sectional nature of the study, we could not delineate the temporal association of these psychosocial factors.

Psychiatric morbidity, maladaptive coping, low social support, and QOL may have negative impact on health outcomes. Therefore, deeper understanding of psychosocial factors on disease progression may help in developing comprehensive intervention strategies for RA.

To conclude, greater disease severity, internalization coping strategy, and lower QOL increase the psychiatric morbidity in patients with RA. Clinician's awareness and interventions to improve $\mathrm{QOL}$ with promoting adaptive coping and timely treatment may further improve their mental health and overall disease course.

Conflict of Interest

None declared. 
122 Psychosocial Profile of People with RA Nebhinani et al.

\section{References}

1 Senra H, Rogers H, Leibach G, et al. Health-related quality of life and depression in a sample of Latin American adults with rheumatoid arthritis. Int J Rheum Dis 2017;20(11):1684-1693

2 Zyrianova Y, Kelly BD, Gallagher C, et al. Depression and anxiety in rheumatoid arthritis: the role of perceived social support. Ir J Med Sci 2006;175(02):32-36

3 Haridoss M, Bagepally BS, Natarajan M. Health-related quality of life in rheumatoid arthritis: systematic review and meta-analysis of EuroQoL (EQ-5D) utility scores from Asia. Int J Rheum Dis 2021r; 24(03):314-326

4 Van der Elst K, De Cock D, Vecoven E, et al; CareRA Study Group. Are illness perception and coping style associated with the delay between symptom onset and the first general practitioner consultation in early rheumatoid arthritis management? An exploratory study within the CareRA trial. Scand J Rheumatol 2016;45 (03):171-178

5 Kilic L, Erden A, Bingham CO III, Gossec L, Kalyoncu U. The reporting of patient-reported outcomes in studies of patients with rheumatoid arthritis: a systematic review of 250 articles. J Rheumatol 2016;43(07):1300-1305

6 Pollack L, Harris R. Measurement of social support. Psychol Rep 1983;53:446

7 Nehra R, Kulhara P, Verma SK. Adaptation of social support questionnaire in Hindi. Indian J Clin Psychol 1996;23:33-39

8 Cooper CL, Faragher EB. Coping strategies and breast disorders/ cancer. Psychol Med 1992;22(02):447-455
9 Sharma Y, Mattoo SK, Kulhara P, Sharma SC, Sharan P. Stress and coping in women with cervical and breast cancer in India. Ger J Psychiatry 2003;2:40-48

10 Saxena S, Chandiramani K, Bhargava RWorld Health Organization Quality of Life. WHOQOL-Hindi: a questionnaire for assessing quality of life in health care settings in India. Natl Med J India 1998;11(04):160-165

11 Folkman S, Moskowitz JT. Coping: pitfalls and promise. Annu Rev Psychol 2004;55:745-774

12 Janiszewska M, Barańska A, Kanecki K, Karpińska A, Firlej E, Bogdan M. Coping strategies observed in women with rheumatoid arthritis. Ann Agric Environ Med 2020;27(03):401-406

13 Bedi GS, Gupta N, Handa R, Pal H, Pandey RM. Quality of life in Indian patients with rheumatoid arthritis. Qual Life Res 2005;14 (08):1953-1958

14 Ibn Yacoub Y, Amine B, Laatiris A, Hajjaj-Hassouni N. Healthrelated quality of life in Moroccan patients with rheumatoid arthritis. Clin Rheumatol 2012;31(10):1471-1477

15 Pitsilka DA, Kafetsios K, Niakas D. Social support and quality of life in patients with rheumatoid arthritis in Greece. Clin Exp Rheumatol 2015;33(01):27-33

16 Larice S, Ghiggia A, Di Tella M, et al. Pain appraisal and quality of life in 108 outpatients with rheumatoid arthritis. Scand J Psychol 2020;61(02):271-280

17 Beşirli A, Alptekin JO, Kaymak D, Özer OA. The relationship between anxiety, depression, suicidal ideation and quality of life in patients with rheumatoid arthritis. Psychiatr Q 2020;91(01):53-64 